\title{
Predicting diabetes mellitus genes via protein-protein interaction and protein subcellular localization information
}

\author{
Xiwei Tang 1,2,3* , Xiaohua Hu ${ }^{2,6^{*}}$, Xuejun Yang ${ }^{3}$, Yetian Fan ${ }^{4}$, Yongfan $\mathrm{Li}^{1}$, Wei Hu${ }^{1}$, Yongzhong Liao ${ }^{1}$, \\ Ming cai Zheng ${ }^{1}$, Wei Peng ${ }^{5}$ and Li Gao ${ }^{6}$
}

From IEEE International Conference on Bioinformatics and Biomedicine 2015

Washington, DC, USA. 9-12 November 2015

\begin{abstract}
Background: Diabetes mellitus characterized by hyperglycemia as a result of insufficient production of or reduced sensitivity to insulin poses a growing threat to the health of people. It is a heterogeneous disorder with multiple etiologies consisting of type 1 diabetes, type 2 diabetes, gestational diabetes and so on. Diabetes-associated protein/gene prediction is a key step to understand the cellular mechanisms related to diabetes mellitus. Compared with experimental methods, computational predictions of candidate proteins/genes are cheaper and more effortless. Protein-protein interaction (PPI) data produced by the high-throughput technology have been used to prioritize candidate disease genes/proteins. However, the false interactions in the PPI data seriously hurt computational methods performance. In order to address that particular question, new methods are developed to identify candidate disease genes/proteins via integrating biological data from other sources.

Results: In this study, a new framework called PDMG is proposed to predict candidate disease genes/proteins. First, the weighted networks are building in terms of the combination of the subcellular localization information and PPI data. To form the weighted networks, the importance of each compartment is evaluated based on the number of interacted proteins in this compartment. This is because the very different roles played by different compartments in cell activities. Besides, some compartments are more important than others. Based on the evaluated compartments, the interactions between proteins are scored and the weighted PPI networks are constructed. Second, the known disease genes are extracted from OMIM database as the seed genes to expand disease-specific networks based on the weighted networks. Third, the weighted values between a protein and its neighbors in the disease-related networks are added together and the sum is as the score of the protein. Last but not least, the proteins are ranked based on descending order of their scores. The candidate proteins in the top are considered to be associated with the diseases and are potential disease-related proteins. Various types of data, such as type 2 diabetes-associated genes, subcellular localizations and protein interactions, are used to test PDMG method.

(Continued on next page)
\end{abstract}

\footnotetext{
*Correspondence: tangxiwei2010@gmail.com; xh29@drexel.edu

${ }^{1}$ School of Information Science and Engineering, Hunan First Normal

University, Changsha, 410205, China

${ }^{2}$ College of Computing and Informatics, Drexel University, Philadelphia, PA

19104, USA

Full list of author information is available at the end of the article
} 
(Continued from previous page)

Conclusions: The results show that the proteins/genes functionally exerting a direct influence over diabetes are consistently placed at the head of the queue. PDMG expands and ranks 445 candidate proteins from the seed set including original 27 type 2 diabetes proteins. Out of the top 27 proteins, 14 proteins are the real type 2 diabetes proteins. The literature extracted from the PubMed database has proved that, out of 13 novel proteins, 8 proteins are associated with diabetes.

\section{Background}

Diabetes mellitus (often also known as diabetes) is a set of metabolic disorders. The latest data from World Health Organization (WHO) (http://www.who.int/diabetes/en/) shows that $9 \%$ of adults worldwide are affected with diabetes. In 2012, 1.5 million people died of the disease. WHO points out that diabetes will become the No. 7 pestilence of threatening the human survival in 2030. It is estimated that America spent $\$ 245$ billion treating diabetes in 2012 (http://www.diabetes.org/). Among these costs, $\$ 176$ billion is directly allocated for medical expenditures, while the remaining funding is used for increasing productivity. Therefore, Diabetes mellitus has evoked great concern in the public health.

In diabetes mellitus, blood sugar levels cannot be reasonably adjusted by the body [1]. For a person with diabetes, the pancreas fails to make sufficient insulin, improperly uses the insulin, or both. In the fast-flowing blood, insulin and glucose work together. The former helps the laster to come into cells of the body and produce energy. Sugar is unable to enter the cells if the insulin does not function properly. This results in the amount of glucose in the blood to go steadily up until generating the high concentration of blood sugar, and causing the cells in the lack of fuel.

Typically, diabetes can be classified into three categories: type 1 diabetes, type 2 diabetes (T2D) and gestational diabetes. When beta cells in the pancreas are destroyed and unable to produce, store, and release the hormone insulin, type 1 diabetes(formerly known as insulin-dependent) occurs [2]. In people with type 1 diabetes, the levels of the blood sugar have not been properly controlled due to the deficient insulin production. The patients with type 1 diabetes often have to regularly inject insulin which help to control their blood sugar. In type 2 diabetes (referred to as non-insulin-dependent), beta cells are able to secrete enough insulin but the body cannot use the insulin effectively and attempts to compensate by making a higher quantity of insulin [3], causing insulin resistance. The production of hepatic glucose cannot be suppressed because of hepatic insulin resistance, and the ability to absorb peripheral glucose is impaired by peripheral insulin resistance. The two factors lead to fasting and postprandial hyperglycemia. The report by World Health Organization (WHO) reveals that $90 \%$ of diabetics worldwide have T2D. In the past three decades the number of persons with T2D has increased sharply in countries of all income levels (http://www.who.int/diabetes/ en/). Gestational diabetes mellitus is a condition where women without prior history of diabetes develop glucose intolerance and high concentration of blood sugar during pregnancy (usually in the third trimester) [4]. Women who had been attacked by gestational diabetes are more likely to develop type 2 diabetes later in life. Diabetes is caused by various factors. The inherited factors, i.e., genetically determined abnormalities of insulin action play an important role. The scope of metabolic abnormalities related to variations of the insulin receptor may cover hyperinsulinaemia and mildly high blood sugar levels to symptomatic diabetes [5-7]. For example, certain mutations of some genes like HLA-DQA1, HLA-DQB1 and HLA-DRB1 raise the risk of causing type 1 diabetes. A few vital proteins in the immune system are generated according to the instructions from these genes [8-10]. Predicting diabetesassociated proteins is very important to understand how diabetes develops since most diabetes-associated variations have an impact on the function of proteins. Linkage studies are often used to determine the genomic intervals which are linked to the disease of interest [11]. Prioritizing a mass of candidate genes via experimental technologies is so expensive and time-consuming that it becomes often impossible to detect the real disease genes by analyzing the list of genes belonged to the interval. Consequently, computational methods have been becoming a prominent option to address such problems.

A lot of computational methods have been developed to sequence and predict the most likely disease-related genes by combining various types of data from different sources, for instance, gene expressing profiles [12, 13], functional annotation information [13-17] and sequence-based features [18]. Meanwhile, huge amounts of protein-protein interactions produced by high-throughput technologies play an important role in the disease identification since they offer functional information in a network environment [19]. Furthermore, the proteins coded by genes which are linked to a specific or familiar disease phenotype tend to stay together and form clusters in the proteinprotein interaction network [20]. In 2006, it was reported that exploiting protein-protein interactions brings prediction of positional candidate disease genes much closer 
to the possibility. A large scale usage of PPIs can predict novel candidate proteins [21]. Many methods and frameworks based on the protein-protein interaction networks have been proposed to rank or identify potential disease candidate genes to understand genetic diseases. For Alzheimer Disease, a list of candidate genes/proteins are prioritized by a computational method in terms of the public human protein-protein interaction networks (PPINs) [22]. In the paper of Erten et al., the topological similarity in the human PPINs is employed to prioritize candidate disease genes [23]. Nevertheless, using the PPINs is a risky choice since false interactions made by high-throughput experiments have a negative impact on the disease gene prioritization [24-28]. In order to mitigate that particular risk, new methods are developed to identify candidate disease genes or proteins via integrating biological data from other sources. In the work of Wu et al., the gene expressing data are integrated with the PPI data to identify cancer-related genes [29]. The functional similarity of Gene Ontology is combined with protein protein interactions (PPIs) to prioritize candidate cardiomyopathy genes [30]. However, these methods neglect the fact that proteins are unable to conduct the desired functions until they take up the correct subcellular compartments. More specifically, a protein can interact with another one only if they are localized at the same subcellular compartments [31,32].

In this article, we propose a method, i.e., Predicting Diabetes Mellitus Genes (PDMG), to rank candidate diabetes mellitus genes by incorporating protein subcellular localization information into the protein-protein interaction networks. First, the protein subcellular localization data are incorporate into the PPINs and the weighted networks are built. Second, we collect the gene records of diabetes from Online Mendelian Inheritance in Man (OMIM) and extract seed genes from these records. Only the genes of T2D are retained since the genes of other diabetes subtypes in OMIM are rare. Then T2D-specific PPINs are constructed by utilizing seed proteins and their interacting neighbors (candidate proteins) from the weighted PPINs. Subsequently, we compute the disease-associated score for each protein in the T2D-specific networks and sort them in descending order. Finally, we discuss the top 27 candidate proteins.

\section{Methods}

In this section, the PDMG method is introduced in detail (see Fig. 1). We first give a general description of sequencing problem of the disease genes. Subsequently, the technology incorporating the subcellular localization information into the PPINs is discussed. Furthermore, we elaborate the method of building disease-specific networks starting from the known disease genes/proteins. Finally, we describe the prioritizing approach of candidate diabetes genes/proteins based on the disease-specific networks.

\section{Disease gene prioritizing problem}

In bioinformatics, the predicting problem of genes that have a close relationship with complex diseases is actually converted into node prioritization problem. The nodes representing the candidate genes/proteins will be scored in accordance with one or more strategies. Then the scores are used to rank them. There is an interesting phenomenon in the biological networks, i.e., the 'guiltby-association' principle. It depends on the assumption that the genes/proteins leading to diseases tend to have the similar or same properties [33]. In term of the principle, people can extract a group of disease-causing genes from the disease databases as the original seed proteins and then quantify the associations between the candidate genes and seed genes. Consequently, the candidate genes are sorted according to the associations $[13,34]$.

Let $D$ indicate a disease of interest. $S$ is a seed gene set in which the genes are associated with $D$. The candidate protein/gene set, represented $C$, is mechanistically associated with $D$. The sets $S$ and $C$ constitute the inputs of the disease gene prioritizing algorithm. The known genes in $S$ related to $D$ serve as a starting point for prioritizing the proteins/genes in $C$. Next, capturing the relationships between the genes in $C$ and the genes in $S$ becomes a critical step. This need to use the topological characteristics of human PPINs. The PPINs, denoted $G=(V, E, w)$, consisting of a group of proteins $V$ and undirected interactions $E$ among the proteins. Meanwhile, $u v \in E$ indicates the interaction between $u \in V$ and $v \in V$. Due to the false positive rate of the protein-protein interaction data, it is necessary to assigned a weight value to each interaction $u v \in E$. The confidence scores represent the reliability of the interactions between $u$ and $v$.

In this article, protein subcellular localization data is used to calculate the confidence scores between proteins. The candidate gene products are sorted based on the scores.

\section{Scoring PPIs}

The eukaryotic cells are elaborately organized into functionally-distinct intracytoplasmic "inclusions" or compartments enclosed within membranes, such as a nucleus and other organelles. The compartments specialize in performing all types of biological functions. The micro-environments have significant influence over protein functions since they control access to and availability of various interacting proteins. In essence, the interactions strongly converge among proteins which are located in the same area of the cells(one-sided binomial test with $P<0.001$ ), but the degree of concentration widely depends on the compartments [35]. For instance, the 


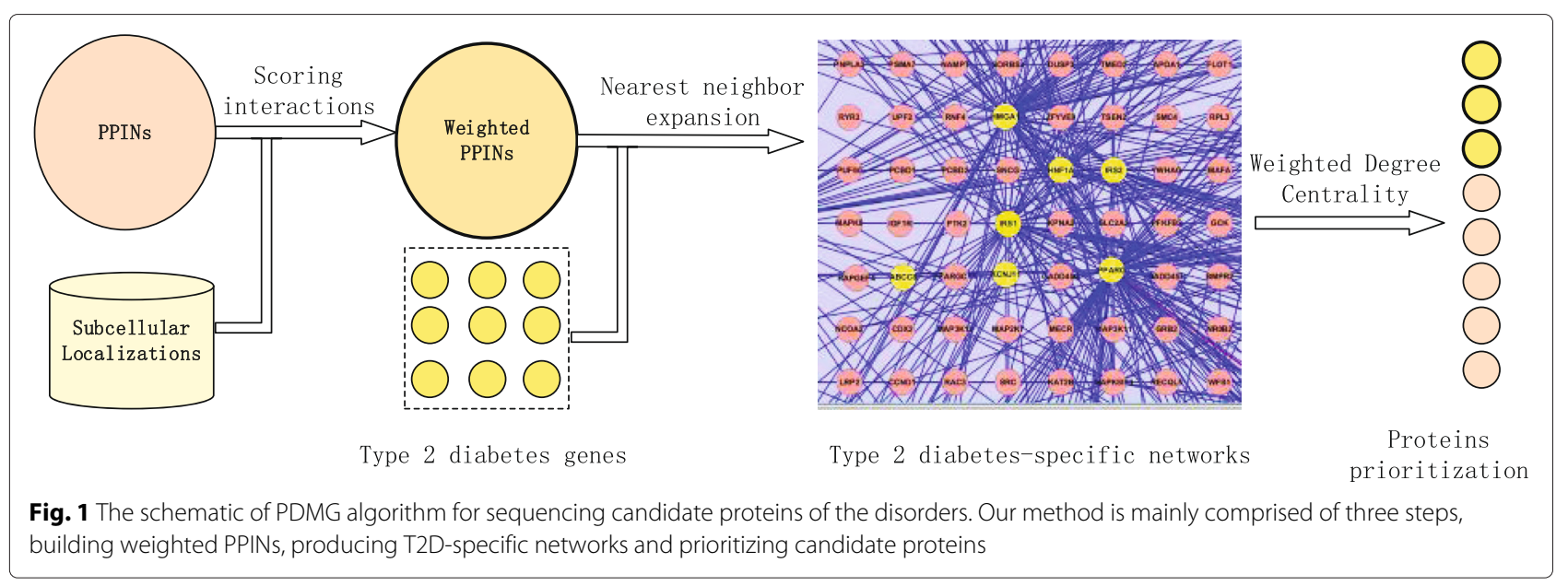

interactions between cytoplasmic proteins are 1.3-fold converged above the threshold. Instead, the interactions among microtubule proteins are 56-fold converged above the threshold. This suggests that the compartment shared by two interacting proteins in the microtubule cytoskeleton better explains the physical and functional interaction than the area of the cytoplasm in which the proteins interact [35]. The fact demonstrates that the significance of different compartments is different in cell activities. After investigating the associations between subcellular localizations and PPIs, Peng et al. find that the farmer is helpful for identifying essential proteins [36, 37]. They give us the motivation of using subcellular localizations to predicting candidate disease genes. Moreover, my research suggests that over half of the T2D genes code essential proteins. Thus, we reason that subcellular localization information can improve the methods of prioritizing candidate disease genes.

Peng et al. report that the significance of a compartment is not out of proportion to the number of interacted proteins in this compartment [36]. In order to score the compartments, the number of the proteins in each compartment is counted. For every compartment, its score is described as the number of interacted proteins in the compartment, denoted by $C_{X}$, divided by the number of proteins in the largest size compartment (consisting of the largest number of interacted proteins), represented by $C_{M}$. The score $S C$ is calculated by using

$$
S C(I)=\frac{C_{X}(I)}{C_{M}}
$$

From the formulation, the value of $S C$ ranges from 0 to 1 , where $I \in\{1,2, \ldots, 11\}$.

According to the scores of compartments, the interactions between proteins in the PPINs can be weighted. The different scores of the compartments mean that some compartment are more important than other ones. The phenomenon leads to the importance of PPIs taken place in different compartments should also be different.

Consider a set of compartments $\operatorname{Loc}(u)$ where protein $u$ is localized. For the two proteins of an interaction $(u, v)$, each protein might be annotated by multiple subcellular localizations. It is reasonable that the interacted protein pairs are localized at the same compartment. Therefore, the interaction $(u, v)$ can be annotated by the shared compartments, i.e., $\operatorname{SLoc}(u, v)=\operatorname{Loc}(u) \bigcap \operatorname{Loc}(v)$. Furthermore, the score of the interaction $(u, v)$ is defined as

$$
W(u, v)=\left\{\begin{array}{l}
\max (S C(I)), \text { if } \operatorname{SLoc}(u, v) \neq \Phi \\
S C\left(C_{N}\right), \text { otherwise }
\end{array}\right.
$$

If $\operatorname{SLoc}(u, v) \neq \Phi$, the score of the interaction $(u, v)$ is assigned with the maximum value of score of the shared compartments. Since the subcellular localization information of some proteins may be missing, for the interactions with $\operatorname{SLoc}(u, v)=\Phi$, the scores of these interactions are assigned with the minimum value of $S C(I)$ among compartments. In Eq. 2, $C_{N}$ is the compartment with the smallest size.

\section{Disease-specific networks}

The OMIM database (http://www.omim.org/) severs as the starting point to extract an initial collection of diseaseassociated genes, i.e., the seed set $S$. With the seed genes and weighted PPIs, we derive a disease-specific networks in terms of the nearest-neighbor expansion approach. In other words, the disease-related networks consist of the seed proteins and their direct neighbors.

\section{Prioritizing candidate disease gene products}

In this subsection, we score the proteins in the diseasespecific networks and rank them based on descending order of their scores. In order to score every candidate protein, we employ the weighted degree centrality (WDC) 
[38], relying on the scored disease-specific networks. Specifically, The score of each candidate disease protein, denoted by $S P D$, is computed in terms of the scored interaction between a protein and its direct neighbor. It can be expressed as

$$
\operatorname{SPD}(u)=\sum_{v}^{N_{u}} W_{u, v}
$$

where $N_{u}$ refers to the set including total neighbors of the protein $u$ and $W_{u, v}$ represents the weighted value of edge between the protein $u$ and its neighbor $v$. All proteins in the disease-specific networks are ranked in descending order of $S P D$.

\section{Results and discussions}

In this section, we evaluate the ability of PDMG to rank candidate disease genes using the known T2Dgene, subcellular localization and PPI information. The datasets used in the experiments are first described. Next, the diabetes-related networks are discussed. Finally, we analyze the novel diabetes genes predicted by PDMG.

\section{Data sources}

Known T2D genes. To form the interaction networks linked to the disease and to detect gene-disease associations from the networks characters, an original set of seed genes known to be associated with the disease is as starting point. We obtain the disease-associated genes of T2D mellitus from OMIM. In OMIM, human genes involved in inherited diseases are recorded in a mini-review format. They are enclosed some information like the gene functions, molecular pathways, and other disease-associated information. To extract a group of T2D-associated genes, we conduct a search of the OMIM database and traverse each OMIM gene record where the term "Diabetes mellitus" is consisted of the "description" field. As a result, 84 OMIM gene records were retrieved. T2D-related entries are shown as Table 1. Based on the HUGO Gene Nomenclature Committee (HGNC) database (http://www.genenames.org/), we replace these genes with their corresponding standard symbols and obtain the seed proteins which correspond to these seed genes. We get 27 proteins coded by the known T2D genes, i.e., GPD2, NEUROD1, IRS1, CAPN10, PPARG, SLC2A2, IGF2BP2, WFS1, CDKAL1, HMGA1,ENPP1, GCK, TCF7L2, KCNJ11, ABCC8, MAPK8IP1, UCP3, MTNR1B, HNF1A, TBC1D4, IRS2, LIPC, HNF1B, GCGR, RETN, AKT2 and HNF4A.

Protein subcellular localizations. The protein subcellular localization data comes from the COMPARTMENTS database [39]. The resource is obtained by integrated a
Table 1 T2D-related gene records

\begin{tabular}{|c|c|c|}
\hline Number & Gene/Locus & Phenotype \\
\hline 1 & Gpd2 & Diabetes, type 2 , susceptibility to \\
\hline 2 & Neurod1 & Diabetes mellitus, noninsulin-dependent \\
\hline 3 & Irs1 & Diabetes mellitus, noninsulin-dependent \\
\hline 4 & Capn10 & Diabetes mellitus, noninsulin-dependent 1 \\
\hline 5 & Pparg & Diabetes, type 2 \\
\hline 6 & Slc2a2 & Diabetes mellitus, noninsulin-dependent \\
\hline 7 & $\operatorname{lgf} 2 \mathrm{bp} 2$ & $\begin{array}{l}\text { Diabetes mellitus, noninsulin-dependent, } \\
\text { susceptibility to }\end{array}$ \\
\hline 8 & Wfs 1 & $\begin{array}{l}\text { Diabetes mellitus, noninsulin-dependent, } \\
\text { association with }\end{array}$ \\
\hline 9 & Cdkal1 & $\begin{array}{l}\text { Diabetes mellitus, noninsulin-dependent, } \\
\text { susceptibility to }\end{array}$ \\
\hline 10 & $\begin{array}{l}\text { Hmga1-rs1, } \\
\text { Hmga1 }\end{array}$ & $\begin{array}{l}\text { Diabetes mellitus, noninsulin-dependent, } \\
\text { susceptibility to }\end{array}$ \\
\hline 11 & Enpp1 & $\begin{array}{l}\text { Diabetes mellitus, non-insulin-dependent, } \\
\text { susceptibility to }\end{array}$ \\
\hline 12 & Gck & $\begin{array}{l}\text { Diabetes mellitus, noninsulin-dependent, } \\
\text { late onset }\end{array}$ \\
\hline 13 & Pax4 & Diabetes mellitus, type 2 \\
\hline 14 & Slc30a8 & $\begin{array}{l}\text { Diabetes mellitus, noninsulin-dependent, } \\
\text { susceptibility to }\end{array}$ \\
\hline 15 & 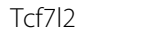 & Diabetes mellitus, type 2, susceptibility to \\
\hline 16 & Kenj11 & Diabetes mellitus, type 2, susceptibility to \\
\hline 17 & Abcc8 & Diabetes mellitus, noninsulin-dependent \\
\hline 18 & Mapk8ip1 & Diabetes mellitus, noninsulin-dependent \\
\hline 19 & Ucp3 & Obesity, severe, and type II diabetes \\
\hline 20 & Mtnr1b & Diabetes mellitus, type 2, susceptibility to \\
\hline 21 & Hnfla & Diabetes mellitus, noninsulin-dependent, 2 \\
\hline 22 & $P d x 1$ & Diabetes mellitus, type II, susceptibility to \\
\hline 23 & Tbc1d4 & Diabetes mellitus, noninsulin-dependent, 5 \\
\hline 24 & Irs2 & Diabetes mellitus, noninsulin-dependent \\
\hline 25 & Lipc & Diabetes mellitus, noninsulin-dependent \\
\hline 26 & Hnf1b & Diabetes mellitus, noninsulin-dependent \\
\hline 27 & Gcgr & Diabetes mellitus, noninsulin-dependent \\
\hline 28 & Retn & $\begin{array}{l}\text { Diabetes mellitus, noninsulin-dependent, } \\
\text { susceptibility to }\end{array}$ \\
\hline 29 & Akt2 & Diabetes mellitus, type II \\
\hline 30 & $\mathrm{Hnf4a}$ & Diabetes mellitus, noninsulin-dependent \\
\hline
\end{tabular}

variety of subcellular localization evidences in terms of high-throughput screens, manually curated annotations and sequence-based identification with automatic text mining for all major model organisms. In the COMPARTMENTS database, the different compartments are labeled as: Nucleus, Golgi apparatus, Cytosol, Cytoskeleton, Peroxisome, Lysosome, Endoplasmic reticulum, Mitochondrion, Endosome, Extracellular space and Plasma membrane. 
Protein-protein interactions. In the experiments, the human protein-protein interactions are downloaded from BioGrid database(Release version BIOGRID-3.2.111) [40]. The human PPINs include 16, 275 proteins and 143, 611 interactions.

\section{T2D-specific networks}

The nearest-neighbor expansion technology is used to construct the T2D-specific protein interaction subnetworks based on the T2D-associated proteins mentioned above and the global PPINs weighted by subcellular localization information. Here, we employ 27 known proteins associated with T2D as the seed diabetes set. The proteins in the weighted PPINs, interacting with the proteins in the seed diabetes set, are pulled out and constitute the candidate T2D protein set. Each interaction between the seed protein and candidate protein composes the diabetesinteraction-set. The two types of proteins (we call them as diabetes-protein-set) and interactions in the diabetesinteraction-set form T2D-specific networks. In the work, the diabetes-protein-set and diabetes-interactionset contains 445 human proteins and 543 interactions, respectively.

\section{Novel proteins predicted by PDMG}

PDMG is used to calculate the relevance score for each protein in the T2D-specific PPINs. We rank them based on descending order of their scores. Table 2 list top 27 T2D candidate proteins containing 14 known T2D-associated proteins and 13 novel proteins. The 13 novel proteins are not initially retrieved from OMIM database based on the term "diabetes mellitus". The results show that our prioritizing technology demonstrates very high specificity: out of 27 top-ranking proteins, 14 proteins are known T2D-related proteins in terms of OMIM annotation. Meanwhile, it can be found that the scores of all known proteins but two ones (HNF1B and GCK) are larger than those of other candidate proteins. Furthermore, to examine PDMG's ability to predict novel diabetes-associated proteins, we use literature study method to determine if the predicted proteins are associated with diabetes. The retrieve results display that out of 13 novel proteins, 8 proteins have been proved to be diabetes-related proteins by literature in the PubMed database (http://www.ncbi.nlm.nih.gov/pubmed). The 8 novel proteins are presented as follows.

\section{CREBBP \#15}

Rende et al. find that CREB binding protein (CREBBP) plays suggestive roles in linking Type 2 diabetes [41]. Their study reveals that heterozygous CREBBP defect leads to raised effects of hormones like leptin and adiponectin, insulin resistance and preventing obesity. Manabe et al. observe that the mRNA expression of CREBBP is reduced in the uteri of ovariectomized STZ-treated diabetic mice [42]. A recent literature report [43] shows that, compared with healthy conditions, the expressing of histone acetyltransferases CREBBP in latent autoimmune diabetes in adults patients is downregulated.

\section{ESR1 \#16}

Linner et al. conclude that the rs2207396 mutation in ESR1 suggests the risk of type 2 diabetes in hypogonadal men [44]. By investigating the relationship between single nucleotide polymorphisms (SNPs) of the candidate gene and the quantitative traits related to metabolic syndrome in Han Chinese type 2 diabetes, Wei et al. [45] find that Rs722208 of ESR1 is associated with fasting plasma glucose $(\mathrm{FPG})(P=0.045)$.

\section{AKT1 \#17}

Devaney et al. [46] report that AKT1 is a risk factor for metabolic syndrome and insulin resistance which is one of the five essential endophenotypes linked to T2D. Hami et al. find a significant bilateral downregulation of AKT1 gene expression in the hippocampus of pups born to diabetic mothers [47].

\section{NRF1 \#18}

By researching defect of Nuclear factor-erythroid 2related Factor 1 (NRF1) in beta-cells, Zheng et al. discover that Nrf1 acts as an essential regulator of mitochondrial function, glucose metabolism and insulin secretion [48]. Specifically, Nrf1 inactivation in beta-cells results in a pre-T2D phenotype because of impairment of insulin secretion and disruption of glucose metabolism [48]. In the study from Hirotsu et al., Nrf1 over-expression has a negative impact on both glucose utilization and production in the liver by suppressing the genes related to both glycolysis and gluconeogenesis [49].

\section{PCBD1 \#19}

The findings from Ferre et al. suggest that a PCBD1 deficiency may cause hypomagnesemia and diabetes [50]. Simaite et al. observe an abundant expression of Pcbd1 in the developing pancreas of both mouse and Xenopus embryos [51]. The genetic evidence obtained by them displays that PCBD1 variations can lead to early-onset nonautoimmune diabetes with characteristics like dominantly inherited HNF1A-diabetes.

\section{YWHAB \#22}

YWHAB interacts with GCGR, a type 2 diabetesrelated protein. To examine the effect of YWHAB on GCGR function, Han et al. investigate glucose production in primary mouse hepatocytes. They discover that YWHAB is overexpressed in mouse hepatocytes. In other words, YWHAB inhibits glucose production [52]. Studies show that YWHAB may plays a critical role in glucose 
Table 2 Top 27 rank-ordered T2D relevant proteins

\begin{tabular}{|c|c|c|c|c|}
\hline Rank & Protein & Score & Description & Diabetes relevance \\
\hline \multirow[t]{2}{*}{1} & PPARG & 85.83 & peroxisome proliferator activated & Known \\
\hline & & & receptor gamma, $T 2 D$, susceptibility to & \\
\hline \multirow[t]{2}{*}{2} & HMGA1 & 63.99 & high mobility group AT-hook 1, Diabetes, & Known \\
\hline & & & noninsulin-dependent, susceptibility to & \\
\hline \multirow[t]{2}{*}{3} & HNF4A & 60.08 & hepatocyte nuclear factor 4 alpha, & Known \\
\hline & & & Diabetes mellitus, noninsulin-dependent & \\
\hline \multirow[t]{2}{*}{4} & IRS1 & 45.12 & insulin receptor substrate 1, Diabetes, & Known \\
\hline & & & noninsulin-dependent & \\
\hline \multirow[t]{2}{*}{5} & HNF1A & 24.21 & HNF1 homeobox A, Diabetes, & Known \\
\hline & & & noninsulin-dependent, 2 & \\
\hline \multirow[t]{2}{*}{6} & AKT2 & 23.28 & v-akt murine thymoma viral & Known \\
\hline & & & oncogene homolog 2, Diabetes, type II & \\
\hline \multirow[t]{2}{*}{7} & TCF7L2 & 20.03 & transcription factor 7 like 2, & Known \\
\hline & & & Diabetes, type 2 , susceptibility to & \\
\hline \multirow[t]{3}{*}{8} & IGF2BP2 & 17.77 & insulin like growth factor 2 & Known \\
\hline & & & mRNA binding protein 2, Diabetes, & \\
\hline & & & noninsulin-dependent, susceptibility to & \\
\hline \multirow[t]{3}{*}{9} & MAPK8IP1 & 14.52 & mitogen-activated protein kinase 8 & Known \\
\hline & & & interacting protein 1, Diabetes, & \\
\hline & & & noninsulin-dependent & \\
\hline \multirow[t]{2}{*}{10} & IRS2 & 12.78 & insulin receptor substrate 2 & Known \\
\hline & & & Diabetes, noninsulin-dependent & \\
\hline \multirow[t]{2}{*}{11} & NEUROD1 & 7.03 & neurogenic differentiation 1 , & Known \\
\hline & & & Diabetes, noninsulin-dependent & \\
\hline 12 & UBC & 6.52 & ubiquitin C & Novel \\
\hline \multirow[t]{2}{*}{13} & HNF1B & 6 & HNF1 homeobox B, Diabetes, & Known \\
\hline & & & noninsulin-dependent & \\
\hline 14 & EP300 & 4 & E1A binding protein $\mathrm{p} 300$ & Novel \\
\hline 15 & CREBBP & 4 & CREB binding protein & Novel \\
\hline 16 & ESR1 & 3.47 & estrogen receptor 1 & Novel \\
\hline \multirow[t]{2}{*}{17} & AKT1 & 3.02 & $v$-akt murine thymoma & Novel \\
\hline & & & viral oncogene homolog 1 & \\
\hline 18 & NRF1 & 3.02 & NFKB repressing factor & Novel \\
\hline 19 & PCBD1 & 3 & pterin-4 alpha-carbinolamine dehydratase 1 & Novel \\
\hline 20 & SP1 & 3 & Sp1 transcription factor & Novel \\
\hline 21 & HDAC4 & 3 & histone deacetylase 4 & Novel \\
\hline \multirow[t]{2}{*}{22} & YWHAB & 2.49 & tyrosine 3-monooxygenase/tryptophan & Novel \\
\hline & & & 5-monooxygenase activation protein beta & \\
\hline 23 & EGFR & 2.47 & epidermal growth factor receptor & Novel \\
\hline \multirow[t]{2}{*}{24} & GCK & 2.45 & glucokinase, Diabetes, noninsulin-dependent, & Known \\
\hline & & & late onset & \\
\hline 25 & ELAVL1 & 2.45 & ELAV like RNA binding protein 1 & Novel \\
\hline 26 & APP & 2.29 & amyloid beta precursor protein & Novel \\
\hline 27 & SUMO2 & 2.01 & small ubiquitin-like modifier 2 & Novel \\
\hline
\end{tabular}


metabolism. YWHAB actually regulates the activity of ChREBP (glucose responsive transcription factor), carbohydrate response element-binding protein, which has important influence on the glucose-mediated induction of proteins associated with hepatic glycolysis and lipogenesis [53]. Besides, YWHAB also controls the activity of AKT, which mediates insulin signaling [54].

\section{EGFR \#23}

Chen et al. suggest that EGFR (epidermal growth factor receptor) mediates TGF-b-induced renal fibrosis and is inhibited by the EGFR inhibitor, erlotinib, in STZinduced diabetic mice [55]. More recently, they also report the resistance of podocyte-specific EGFR knockout mice to the development of diabetes-associated podocyte damage [56].

\section{SUMO2 \#27}

The transcriptional activity of $\mathrm{T}$ and $\mathrm{B}$ cells is negatively regulated by the mouse SUMO2 [57, 58]. The mouse SUMO2 in T cells is overexpressed, which inhibits the production of both Th1 and Th2 cytokines $[57,58]$. This means that the mouse SUMO2 plays a more complex role in the progression of autoimmune diabetes. The early literature [59] also shows that SUMO is related to NF-kB activation and may thus be linked to type 1 diabetes with apoptosis in pancreatic beta cells.

\section{Conclusions}

With the available PPI data increasing rapidly, a unprecedented opportunity for predicting disease-associated genes/proteins at the network level is appear. The PPINs have been widely adopted by many state of the art algorithms to address the gene prioritization problem. They are based on the principle that the genes/proteins causing similar diseases tend to cluster together in the network. However, the high false positive rates and false negative rates of the available PPI data have a negative influence on the accuracy of methods identifying disease genes/proteins only by the topological properties of the networks. To improve the prediction, researchers develop all kinds of new approaches to predict candidate disease genes via combining other data from different sources with PPINs. But these methods neglect an obvious fact proteins don't perform their desired functions unless they are localized at the appropriate subcellular compartments. In this work, subcellular localization data are integrated with PPINs. The combination is achieved by building disease-specific PPINs and employing them in the prioritization. Specifically, OMIM is used to obtain seed genes/proteins of type 2 diabetes. With these seed proteins, we produce T2D-specific PPINs from the weighted PPINs based on the nearest-neighbor expansion approach. And then the scores of candidate T2D proteins are calculated by WDC method. Finally, we rank the proteins based on descending order of their scores.

In order to prove PDMG's ability to predict potential disease-related proteins, we employ the literature review method to analyze the novel proteins/genes predicted by PDMG. The results show that PDMG has predicted 13 novel proteins in top 27 candidate proteins. Out of the 13 novel proteins, 8 proteins CREBBP, ESR1, AKT1, NRF1, PCBD1, YWHAB, EGFR, SUMO2 are associated with diabetes in literature. The evidences display that the 8 novel proteins are recovered from the interaction data and subcellular localization information analysis although they are not retrieved from OMIM database. Therefore, PDMG method can make up for the false negatives (to an extent) of PPINs. Besides, according to the ranked candidate proteins, one may gain many new biological suppositions about the new protein functions in the context of protein interaction networks out of scope of this work.

\section{Acknowledgements}

Publication of this article was partially funded by the National Natural Science Foundation of China under Grant Nos. 61472133, 61502214, 31560317, Hunan Provincial Natural Science Foundation of China Nos. 15JJ2038, 15JJ2037, Research Foundation of Education Bureau of Hunan Province Nos. 14A027, [2015]118, [2013]532, Hunan Key Laboratory no. 2015TP1017.

\section{Declarations}

This article has been published as part of BMC Genomics Vol 17 Suppl 42016 : Selected articles from the IEEE International Conference on Bioinformatics and Biomedicine 2015: genomics. The full contents of the supplement are available online at http://bmcgenomics.biomedcentral.com/articles/ supplements/volume-17-supplement-4.

\section{Authors' contributions}

$X T$ constructs PDMG framework, carries out the corresponding algorithm and writes the article. $X H$ and $X Y$ conceived of the study. $Y L$, YF and $Y L$ provide a number of valuable suggestions in development of the algorithm. WP and WH perform the data collection and analysis. All authors read and approved the final manuscript.

\section{Competing interests}

The authors declare that they have no competing interests.

\section{Author details}

${ }^{1}$ School of Information Science and Engineering, Hunan First Normal University, Changsha, 410205, China. ${ }^{2}$ College of Computing and Informatics, Drexel University, Philadelphia, PA 19104, USA. ${ }^{3}$ College of Computer, National University of Defense Technology, Changsha, 410073, China. ${ }^{4}$ School of

Mathematical Sciences, Dalian University of Technology, Dalian, 116023, China. ${ }^{5}$ Computer Center, Kunming University of Science and Technology, Kunming, 650500, China. ${ }^{6}$ School of Computer, Central China Normal University, Hubei, 430079, China.

Published: 18 August 2016

\section{References}

1. Mellitus D. Diagnosis and classification of diabetes mellitus. Diabetes care. 2005;28(S37):S5-S10.

2. Davies JL, Kawaguchi $Y$, Bennett ST, et al. A genome-wide search for human type 1 diabetes susceptibility genes. Nature. 1994;371 (6493): 130-6.

3. Butler AE, Bonner-Weir S, et al. Janson, J. Diabetes. 2003;52(1):102-10.

4. Buchanan TA, Xiang AH. Gestational diabetes mellitus. J Clin Invest. 2005;115(3):485-91. 
5. Marx J. Unraveling the causes of diabetes. Science. 2002;296(5568):686.

6. Notkins AL. The causes of diabetes. Sci Am. 1979;241(5):62.

7. Loeken MR. Advances in understanding the molecular causes of diabetes-induced birth defects. J Soc Gynecologic Invest. 2006;13(1):2-10.

8. Nguyen C, Varney MD, Harrison LC, et al. Definition of high-risk type diabetes HLA-DR and HLA-DQ types using only three single nucleotide polymorphisms. Diabetes. 2013;62(6):2135-40.

9. HuX, Deutsch AJ, Lenz TL, et al. Additive and interaction effects at three amino acid positions in HLA-DQ and HLA-DR molecules drive type 1 diabetes risk. Nat Genet. 2015;47(8):898-905.

10. Chen LM. Association of the HLA-DQA1 and HLA-DQB1 Alleles in Type 2 Diabetes Mellitus and Diabetic Nephropathy in the Han Ethnicity of China. Exp Diabetes Res. 2013;2013:1-5.

11. Glazier AM, Nadeau JH, Aitman TJ. Finding Genes That Underlie Complex Traits. Science. 2002;298(5602):2345-9.

12. Lage $K$, Karlberg E, et al. A human phenome-interactome network of protein complexes implicated in genetic disorders. Nat Bio. 2007;25(3): 309-16.

13. Aerts $S$, Lambrechts $D$, et al. Gene prioritization through genomic data fusion. Nat Biotech. 2006;24(5):537-44.

14. Adie $E$, Adams R, et al. SUSPECTS:enabling fast and effective prioritization of positional candidates. Bioinformatics. 2006;22(6):773-4.

15. Turner F, Clutterbuck D, Semple C. POCUS: mining genomic sequence annotation to predict disease genes. Genome Biology. 2003;4(11):R75.

16. Masotti D, Nardini C, et al. TOM: enhancement and extension of a tool suite for in silico approaches to multigenic hereditary disorders. Bioinformatics. 2008;24(3):428-9.

17. Chen J, Bardes EE, et al. ToppGene Suite for gene list enrichment analysis and candidate gene prioritization. Nucleic Acids Res. 2009;37(suppl 2): W305-11.

18. Adie EA, Adams RR, et al. Speeding disease gene discovery by sequence based candidate prioritization. BMC Bioinformatics. 2005;6(55):1-13.

19. Stelzl U, Wanker EE. The value of high quality protein-protein interaction networks for systems biology. Curr Opin Chem Biol. 2006;10:551-8.

20. Gandhi TKB, Zhong J, et al. Analysis of the human protein interactome and comparison with yeast, worm and fly interaction datasets. Nat Genet. 2006;38:285-93.

21. Oti M, Snel B, Huynen MA, et al. Predicting disease genes using proteinCprotein interactions. J Med Genet. 2006;43(8):691-8.

22. Chen JY, Shen C, Sivachenko AY. Mining Alzheimer disease relevant proteins from integrated protein interactome data. Pac Symp Biocomput. 2006;11:367-78.

23. Erten $\mathrm{S}$, Bebek $\mathrm{G}$, et al. Disease gene prioritization based on topological similarity in protein-protein interaction networks. Res Comput Mol Biol. 2011;2011:54-68.

24. Sprinzak E, Sattath S, Margalit H. How Reliable are Experimental Protein-Protein Interaction Data J Mol Biol. 2003;327(5):919-23.

25. Chen J, Yuan B. Detecting Functional Modules in the Yeast Protein-Protein Interaction Network. Bioinformatics. 2006;22(18):2283-90

26. Bader GD, Hogue CWV. Analyzing yeast protein-protein interaction data obtained from different sources. Nat Biotechnol. 2002;20(10):991-7.

27. Batada N, Hurst LD, Tyers M. Evolutionary and physiological importance of hub proteins. PLoS Comp Bio. 2006;2:e88.

28. Collins SR, Kemmeren P, Zhao XC, et al. Toward a comprehensive atlas of the physical interactome of Saccharomyces cerevisiae. Mol Cell Proteomics. 2007:6(3):439-50.

29. Wu C, Zhu J, Zhang X. Integrating gene expression and protein-protein interaction network to prioritize cancer-associated genes. BMC Bioinformatics. 2012;13(1):182.

30. LiW, Chen L, He W, et al. Prioritizing Disease Candidate Proteins in Cardiomyopathy-Specific Protein-Protein Interaction Networks Based on "Guilt by Association" Analysis. PloS one. 2013;8(8):e71191.

31. Kumar A, Agarwal S, et al. Subcellular localization of the yeast proteome. Genes and development. 2002;16(6):707-19.

32. de Lichtenberg $U$, Jensen $\sqcup$, et al. Dynamic complex formation during the yeast cell cycle. science. 2005;307(5710):724-7.

33. Altshuler D, Daly M, Kruglyak L. Guilt by association. Nat Genet. 2000;26(2):135-8.

34. Kohler $\mathrm{S}$, Bauer $\mathrm{S}$, Horn D, et al. Walking the interactome for prioritization of candidate disease genes. Am J Hum Genet. 2008;82(4):949-58.
35. Huh WK, Falvo JV, et al. Global analysis of protein localization in budding yeast. Nature. 2003;425(6959):686-91.

36. Peng $X$, Wang J, et al. An efficient method to identify essential proteins for different species by integrating protein subcellular localization information. Bioinformatics Biomed. 2015;2015:277-80.

37. Peng $X$, Wang J, et al. Rechecking the Centrality-Lethality Rule in the Scope of Protein Subcellular Localization Interaction Networks. PloS one. 2015;10(6):1-22.

38. Tang $X$, Wang J, et al. Predicting essential proteins based on weighted degree centrality. IEEE/ACM Trans Comput Biol Bioinformatics. 2014;11(2): 407-18.

39. Binder JX, Pletscher-Frankild S, et al. COMPARTMENTS: unification and visualization of protein subcellular localization evidence. Database. 2014;2014: bau012.

40. Stark C, Breitkreutz BJ, et al. Biogrid: a general repository for interaction datasets. Nucleic Acids Res. 2006;34(1):D535—9.

41. Rende D, Baysal N, Kirdar B. Complex disease interventions from a network model for type 2 diabetes. PloS One. 2013;8(6):e65854.

42. Manabe $Y$, Tochigi $M$, et al. Insulin-like growth factor 1 mRNA expression in the uterus of streptozotocin-treated diabetic mice. J Reprod Dev. 2013;59(4):398-404.

43. Liu X, Xu J. Reduced Histone H3 Acetylation in CD4. Disease Markers. 2015;2015:1-8.

44. Linner C, Svartberg J, Giwercman A, et al. Estrogen receptor alpha single nucleotide polymorphism as predictor of diabetes type 2 risk in hypogonadal men. Aging Male. 2013;16(2):52-7.

45. Wei FJ, Cai CY, et al. Quantitative candidate gene association studies of metabolic traits in Han Chinese type 2 diabetes patients. Genet Mol Res GMR. 2015;14(4):15471.

46. Devaney JM, Gordish-Dressman H, et al. AKT1 polymorphisms are associated with risk for metabolic syndrome. Hum Genet. 2011;129(2): 129-39.

47. Hami J, Kerachian MA, et al. Effects of streptozotocin-induced type 1 maternal diabetes on PI3K/AKT signaling pathway in the hippocampus of rat neonates. J Receptors Signal Transduction. 2015;2015:1-7.

48. Zheng H, Fu J, et al. CNC-bZIP protein Nrf1-dependent regulation of glucose-stimulated insulin secretion. Antioxidants Redox Signal. 2015;22(10):819-31.

49. Hirotsu Y, Higashi C, et al. Transcription factor NF-E2-related factor 1 impairs glucose metabolism in mice. Genes Cells. 2014;19(8):650-65.

50. Ferre S, de Baaij JHF, et al. Mutations in PCBD1 cause hypomagnesemia and renal magnesium wasting. J Am Soc Nephrol. 2013;2013:. ASN 2013040337.

51. Simaite $D$, Kofent $J$, et al. Recessive mutations in PCBD1 cause a new type of early-onset diabetes. Diabetes. 2014;63(10):3557-64.

52. Han J, Zhang M, et al. The Identification of Novel Protein-Protein Interactions in Liver that Affect Glucagon Receptor Activity. PloS one. 2015;10(6):e0129226.

53. Sakiyama H, Wynn RM, et al. Regulation of Nuclear Import/Export of Carbohydrate Response Element-binding Protein (ChREBP) INTERACTION OF AN alpha-HELIX OF ChREBP WITH THE 14-3-3 PROTEINS AND REGULATION BY PHOSPHORYLATION. J Biol Chem. 2008;283(36): 24899-908.

54. Somanath PR. 14-3-3 beta-Rac1-p21 activated kinase signaling regulates Akt1-mediated cytoskeletal organization, lamellipodia formation and fibronectin matrix assembly. J Cell Physiol. 2009;218(2):394-404.

55. Chen J, Chen JK, et al. EGFR signaling promotes TGF-dependent renal fibrosis. J Am Soc Nephrol. 2012;23(2):215-24.

56. Chen J, Chen JK, Harris RC. EGF receptor deletion in podocytes attenuates diabetic nephropathy. J Am Soc Nephrol. 2015;26(5):1115-25.

57. Hwang KW, Won TJ, et al. Erratum to "Characterization of the regulatory roles of the SUMO". Diabetes/metabolism Res Rev. 2012;28(2):196-202.

58. Hwang KW, Won TJ, et al. Characterization of the regulatory roles of the SUMO. Diabetes/metabolism Res Rev. 2011;27(8):854-61.

59. Owerbach D, Pina L, Gabbay KH. A 212-kb region on chromosome $6 q 25$ containing the TAB2 gene is associated with susceptibility to type 1 diabetes. Diabetes. 2004;53(7):1890-3. 